Annuaire suisse de politique de développement

15 | 1996

Annuaire Suisse - Tiers Monde 1996

\title{
Clauses sociales au service d'une économique mondiale équitable
}

Dix thèses d'éthique économique

\section{Christoph Stückelberger}

\section{(2) OpenEdition}

\section{Journals}

Édition électronique

URL : http://journals.openedition.org/aspd/1522

DOI : $10.4000 /$ aspd. 1522

ISSN : 1663-9669

\section{Éditeur}

Institut de hautes études internationales et du développement

Édition imprimée

Date de publication : 1 mars 1996

Pagination : 239-246

ISSN : 1660-5934

\section{Référence électronique}

Christoph Stückelberger, «Clauses sociales au service d'une économique mondiale équitable », Annuaire suisse de politique de développement [En ligne], 15 | 1996, mis en ligne le 16 mai 2013, consulté le 08 septembre 2020. URL : http://journals.openedition.org/aspd/1522 ; DOI : https:// doi.org/10.4000/aspd. 1522 


\title{
Clauses sociales au service d'une économique mondiale équitable
}

\section{Dix thèses d'éthique économique}

\author{
Christoph Stückelberger
}

"La communauté analysera de plus en plus souvent la situation du point de vue de ce qu'elle apporte au plus grand nombre. La liberté incontrôlée de l'individu, qui en fin de compte asservit des peuples entiers, fera progressivement place à la liberté du plus grand nombre. L'Etat édictera notamment une loi sur la police dans les fabriques... L'Etat cherchera enfin à établir une loi internationale. $\mathrm{Ce}$ n'est que lorsqu'une loi édictée par tous les pays industrialisés, ou en tout cas par les plus importants - et dès lors considérée comme norme contraignante pour toute législation particulière - imposera la formation scolaire obligatoire pour les enfants, empêchera l'embauche de jeunes enfants et l'exploitation sans scrupule de leur force de travail qui s'exerce au détriment de leur développement physique et intellectuel, interdira les travaux inhumains et prendra en charge le travailleur malade, âgé et brisé, que ces phénomènes disparaîtront dans les fabriques."

Cette citation n'est pas tirée d'un discours prononcé dans le cadre des négociations du GATT des dernières années mais d'un ouvrage intitulé "Ein Wort über die Fabrikindustrie" datant de 1858. ${ }^{1}$ L'auteur, Bernhard Becker, était pasteur dans le petit canton montagnard de Glaris, en Suisse, où $55 \%$ de la population active travaillait dans l'industrie du coton. La famine régnait et nombre de familles - des réfugiés économiques en quelque sorte - durent émigrer

1. Becker, B: Ein Wort über die Fabrikindustrie (1858). Avec une introduction de H.-U. Schiedt, édité par l'institut d'éthique sociale de la SEK, Berne 1990. 
en Amérique. Becker fut l'un des initiateurs de la loi sur les fabriques du canton de Glaris. Cette loi, la première du genre en Suisse et même en Europe, limitait la journée de travail à 12 heures. II y a 140 ans, le pasteur Becker plaidait déjà pour des législations nationales et internationales visant à régler les rapports sociaux et à instaurer la justice sociale dans l'industrie.

C'est ainsi qu'est né un mouvement de longue haleine vers une économie de marché sociale, un mouvement profondément imprégné d'éthique protestante. ${ }^{2}$ Aujourd'hui, le débat pour le maintien (ou l'obtention) d'un partage socialement équitable des richesses dégagées par l'économie de marché a rebondi au niveau tant national qu'international suite à la libéralisation du marché mondial. Dans le monde entier, le respect de normes sociales minimales dans le domaine du travail, par le biais de clauses sociales, est un aspect important de la lutte pour une existence plus digne. Dans ces thèses, nous entendons par clauses sociales les dispositions réglant les normes minimales en matière sociale (et en droit du travail) en vigueur sur le marché international et permettant de prendre des mesures positives et négatives à l'encontre de ceux qui ne respectent pas ces normes minimales.

Nous avons énoncé sous forme résumée dix principes éthiques devant régir ces clauses sociales.

\section{Le but de l'économie est d'assurer une existence digne pour tous}

Quelle est la finalité de l'activité économique? II faut constamment revenir à cette question décisive. Accroissement du capital, croissance économique et victoire sur la concurrence ne sont pas des fins en soi mais tout au plus des moyens permettant d'atteindre un objectif. Mais quel objectif? Tous les hommes, toutes les créatures vivantes, ont le même besoin fondamental de vivre, d'évoluer et de s'épanouir. En utilisant un langage théologique, Dieu leur a fait don d'une vie digne. La finalité de l'activité économique est donc de permettre au plus grand nombre possible d'êtres humains et de créatures vivantes de mener une existence digne en produisant et en partageant des produits et des prestations. L'économie vise donc à garantir une vie digne dans le monde entier, une notion qui va donc bien au-delà de l'augmentation du produit national brut, comme en témoigne notamment le «Human Developement Index» HDI du programme des Nations Unies pour le développement (PNUD) ou des indices comme celui de Daly/Cobb. ${ }^{3}$

2. cf. Brakelmann, G./Jähnichen, T.: Die protestantischen Wurzeln der sozialen Marktwirtschaft. Ein Quellenband. Gütersloh, 1994. La thèse centrale de cet ouvrage est que le concept d'économie sociale de marché est fondamentalement imprégné des traditions d'éthique sociale du protestantisme. (Introduction, 13)

3. PNUD: Human Development Report, 1994, 90-226. Daly, H/Cobb, J.B.: For the Common Good: Redirecting the Economy Toward Community, the Environment and a Sustainable Future, Boston 1989. 


\section{Les Droits de l'homme constituent le fondement du développement social}

Malgré les discussions sur leur valeur universelle, la Déclaration universelle des Droits de l'homme et les pactes de l'ONU sur les droits politiques, économiques, sociaux, culturels et le «droit au développement» constituent la référence unanimement reconnue dans le monde entier pour évaluer si le développement social se fait dans la dignité. Le développement économique et le commerce mondial - deux aspects importants du développement social - doivent donc être évalués à l'aune de ce critère. Chaque fois qu'une mesure économique est prise, il faut donc se demander à quel point elle favorise ou au contraire entrave un développement social garantissant la dignité et le respect des Droits de I'homme. Les clauses sociales doivent par ailleurs accorder une importance particulière aux droits de la femme car ceux-ci font partie intégrante des Droits de l'homme.

Les clauses sociales ont pour but de lutter contre toute forme de violation des Droits de l'homme dans le monde. Les sept conventions de l'OIT visant à créer une clause sociale se basent sur les Droits de l'homme fondamentaux. Six d'entre elles (le travail des enfants faisant exception) ont été ratifiées par 110 à 135 des 193 Etats que compte la Terre.

Le lien entre la régulation de l'économie mondiale et la responsabilité de respecter les Droits de l'homme est toujours remis en question en pratique car on craint a) qu'il entraîne des préjudices en matière de concurrence, b) que différents Droits de l'homme ne soient contradictoires (par exemple le droit au développement et l'interdiction du travail des enfants) et c) que les clauses sociales ne comprennent que des normes minimales limitées à des domaines précis. On peut répondre à ces craintes en affirmant que a) les Droits de l'homme sont inaliénables et que, d'un point de vue éthique, leur respect ne peut donc pas être soumis à l'opportunité économique ou politique, b) qu'en cas de conflit entre différents droits, il convient de procéder à une évaluation du problème en fonction de critères éthiques et $c$ ) que les Droits de l'homme sont certes indivisibles mais que cela n'est pas une raison suffisante pour s'abstenir de protéger certains droits spécifiques par le biais de clauses sociales (à condition que ces mesures ne soient pas érigées en valeurs absolues).

\section{La cohérence politique passe par une coresponsabilité à tous les niveaux}

Les activités humaines ne sont jamais totalement exemptes de contradictions, qu'il faut toutefois essayer de limiter le plus possible. A cet effet, il convient d'harmoniser politique des Droits de l'homme et politique économique extérieure, politique extérieure et politique sociale, politique de l'environnement et politique économique, etc... pour aboutir à une politique générale cohérente. En d'autres termes, la politique commerciale pratiquée par une multinationale dans un pays en développement doit être la même que celle qu'elle met en oeuvre 
dans son pays d'origine. Le respect de normes sociales minimales régissant les activités économiques tant nationales qu'internationales est l'expression et la condition d'une action cohérente. Cette cohérence est une priorité tant au niveau national ${ }^{4}$ qu'au niveau des relations multilatérales ${ }^{5}$ et de l'éthique des entreprises.

\section{La justice doit viser à réduire le fossé économique entre les pau- vres et les nantis}

L'éthique économique mesure toujours l'activité économique en fonction du critère de justice. Reste à définir ce qui est juste. Nous n'entrerons pas ici dans le vaste débat théologique et éthico-économique sur la conception de la justice, si ce n'est pour souligner deux principes de justice de John Rawls devenus célèbres et aujourd'hui largement répandus en éthique économique. Le premier est formulé comme suit: "Tout le monde jouit du même droit de disposer du système le plus vaste de mêmes libertés fondamentales accessibles à tous"; ${ }^{6}$ les accords du GATT/OMC, conçus comme un système garantissant les mêmes libertés commerciales dans le monde entier, s'en inspirent. ${ }^{7} \mathrm{Ce}$ premier principe est toutefois indissociable du second: "Les inégalités sociales et économiques doivent être réglées de la manière suivante: a) elles doivent apporter le plus grand avantage possible aux plus défavorisés..." ${ }^{8}$ En simplifiant les choses, est juste ce qui protège et renforce les plus faibles.

Dans une perspective économique, le commerce international se nourrit des différences de coûts de production (matières premières, salaires, standards sociaux, etc...) qui existent entre différents lieux de production. II doit toutefois aussi essayer de combler ces différences s'il entend prendre au sérieux les critères d'un développement social garantissant la dignité dont nous avons parlé ci-dessus.

4. C'est ainsi que la politique extérieure officielle de la Suisse souhaite prôner "la cohérence... entre politique intérieure et extérieure,... entre différents domaines de la politique extérieure,... entre les objectifs et les moyens de la politique extérieure..." (rapport du Conseil fédéral sur la politique extérieure de la Suisse dans les années 90, 29 novembre 1993, 45). On trouve aussi des propositions pour une politique suisse cohérente en matière de développement chez Gerster, R.: Nord-Süd Politik: abschreiben oder investieren? Perspektiven der schweizerischen Entwicklungszusammenarbeit, Zurich, 1995, 63-68.

5. cf. notamment Gemeinsame Konferenz Kirche und Entwicklung (GKKE) / European Ecumenical Organisation for Development (EECOD): Towards Coherence in North/ South Policy: the Role of the European Union ${ }_{\perp}$ compte-rendu d'un séminaire de Bruxelles, 19-21 janvier 1994.

6. Rawls, J.: Eine Theorie der Gerechtigkeit, Francfort a.M. 1979, 336.

7. Rawls applique ce principe d'abord aux libertés politiques en démocratie (ibidem 223-290), ensuite à l'économie politique (ibidem 306 ss. notamment).

8. Ibidem, 336 . 


\section{La protection des faibles ne doit pas être négligée pour préserver des avantages locaux}

Les clauses sociales sont combattues par les gouvernements des pays du Sud sous prétexte qu'ils perdraient l'avantage que leur confère leur main-d'oeuvre bon marché sur le marché international. Cette position leur sert souvent d'excuse pour défendre fallacieusement les intérêts de la classe des nantis au détriment des plus pauvres. A l'inverse, les gouvernements et les entreprises des pays du Nord approuvent les clauses sociales, parfois avec l'arrière-pensée protectionniste ${ }^{9}$ de défendre les intérêts des pays riches au détriment des pays économiquement faibles.

La question fondamentale est de savoir si les avantages liés au lieu de production profitent au plus grand nombre ou à quelques privilégiés, car le choix du lieu ne doit pas pénaliser les plus faibles. D'un autre côté, la protection de ces derniers n'implique pas la disparition de la concurrence, qui élimine les protagonistes économiques les moins capables et les moins performants. Comme vient de le réaffirmer avec raison l'économiste Hermann Sautter, solidarité et concurrence ne s'excluent pas. ${ }^{10}$

\section{La liberté ne peut se définir que par rapport à la justice}

On attend de l'ouverture des marchés qu'elle dynamise l'économie mondiale. Cette mesure a pour effet de remettre la liberté à la première place des valeurs économiques. La liberté est certes une valeur fondamentale importante mais toute valeur porte en elle des germes de perversion lorsqu'on en fait un absolu. Or, la liberté érigée en absolu débouche sur le "tout est permis" et sur l'écrasement des faibles tout comme la justice érigée en absolu glisse vers le totalitarisme et la terreur. Les valeurs fondamentales ne restent humaines que si elles demeurent en équilibre les unes par rapport aux autres. La liberté combinée avec la justice permet un développement harmonieux de l'humanité. De même, la justice autorise aussi un développement social garant de la dignité humaine pour autant qu'elle n'étouffe pas la liberté. Jusqu'à présent, la libéralisation du commerce mondial n'a pas réussi à réduire les inégalités et les injustices du monde économique. C'est pourquoi le marché mondial libéralisé a besoin d'être encadré par des normes sociales minimales.

9. Des études économiques montrent que le chômage dans les pays du Nord est moins dû aux délocalisations dans les pays du Sud qu'aux progrès technologiques. II faut donc considérer avec prudence l'hypothèse selon laquelle les clauses sociales auraient des effets protectionnistes et permettraient de sauvegarder des emplois dans le Nord.

10. Sautter, H.: Moralisches Urteil und ökonomisches Kalkül, in "Zeitschrift für Evangelische Ethik» 39, 1995, 132-136. 


\section{Le développement économique ne peut se définir que par rapport au développement social}

Les adversaires des clauses sociales avancent toujours l'argument selon lequel il serait plus efficace de libéraliser que d'entraver le marché si l'on veut aboutir à un progrès social;"11 ils prétendent aussi que la croissance économique conditionne le développement social et le financement de l'Etat social. Encore faut-il déterminer quels facteurs conditionnent le développement économique et quels autres découlent de ce dernier. Les études économiques empiriques qui s'intéressent à cette question aboutissent à des conclusions ${ }^{12}$ très diverses mais il semble bien que le développement social soit à la fois la condition sine qua non à un développement économique durable (au sud comme au nord) et une conséquence de ce développement. De nombreuses études montrent en effet que le développement social augmente la productivité, l'efficacité et l'image de l'économie, que des salaires supérieurs et l'émergence de classes moyennes stimulent le pouvoir d'achat, qu'une meilleure formation augmente la compétitivité, que le partenariat social renforce la stabilité sociale et politique, que la répartition équitable des terres entre de nombreux cultivateurs et le droit pour la femme de posséder elle-même de la terre est également bénéfique pour la productivité. On peut donc supposer que, loin de la restreindre, les clauses sociales (et écologiques) augmentent la compétitivité de l'économie à moyen terme en tout cas. Les clauses s'inscrivent donc non seulement dans une logique des droits de l'homme mais dans une logique économique.

\section{Les prix doivent refléter la réalité écologique et sociale}

"Les prix doivent refléter la réalité". Ce postulat d'E.U. von Weizsäcker est aujourd'hui reconnu par ceux qui s'occupent de politique - et d'éthique - de l'environnement mais il n'est pas encore appliqué dans la pratique, tant s'en faut! Considérés jusqu'ici le plus souvent comme coûts externes que la communauté devrait prendre en charge, les coûts écologiques d'un produit (qui intègrent l'utilisation des ressources, la gestion des déchets et les dommages immédiats) doivent désormais être internalisés. Or, les prix actuels ne correspondent qu'au cinquième ou au dixième de la réalité écologique. II en va de même de nombreux coûts sociaux, surtout au Sud mais partiellement aussi au Nord. II importe que les prix traduisent les coûts sociaux véritables. Les problè-

11. Grossmann, H./Koopmann, G.: Sozialstandards im internationalen Handel?, in "Wirtschaftsdienst", nov. 1994, 585-591 (591).

12. Les résultats empiriques doivent être analysés selon des critères d'éthique économique, ce que nous ne pouvons qu'esquisser ici: cf. par ex. OCDE: Trade and Labor Standards. A Review of the Issues, Paris, mai 195; Wood, A.: North-South Trade, employment and inequality, changing fortunes in a skill-driven world, Oxford 1994; Charnovitz, St.: The Influence of International Labor Standards on the World Trading Regime: A Historical Overview, International Labor Review, sept-oct. 1987. 
mes de santé occasionnés par le travail sont considérés comme des coûts de santé et à ce titre, ils sont à la charge de l'Etat (ce qui correspond au niveau écologique à la surexploitation des ressources, à la différence près qu'il s'agit ici de ressources humaines); par ailleurs, le fait d'empêcher les travailleurs de suivre une bonne formation élargit le fossé qui sépare les pays en développement des pays industrialisés en ce qui concerne l'accès au savoir-faire (ce qui - au niveau écologique - correspond à l'absence de prévoyance pour les générations futures). Les coûts sociaux doivent notamment comprendre aussi les coûts spécifiques au sexe. Les femmes subissent en effet les coûts sociaux plus lourdement que les hommes (double fonction de travailleur et de mère, salaires inférieurs, responsabilité de la santé de la famille, etc...).

\section{II convient de créer les instruments juridiques nécessaires pour imposer des normes économiques minimales acceptables du point de vue éthique}

Comment ancrer dans le droit des normes d'éthique économique comme on le ferait pour des normes sociales minimales? La palette des moyens appliqués est vaste. L'éducation éthique et le travail de persuasion, qui passent par la prise de conscience et le choix d'arguments judicieux, sont des moyens fondés sur la coopération et la participation qui correspondent à l'image chrétienne de l'Homme aimant son prochain et le respectant comme un partenaire à part entière. Une telle forme de coopération peut déboucher sur le respect spontané des normes comme sur la création de lois communes. Une éthique fondée sur la justice, la protection du faible, etc... n'a pas forcément besoin du droit mais elle peut aussi s'appuyer sur lui.

Au niveau national, on sait depuis longtemps que les normes éthiques qui régissent les rapports contractuels commerciaux doivent être ancrées dans le droit. Or, sur un marché mondial marqué par une interpénétration croissante des marchés nationaux, il existe d'importantes lacunes à ce niveau. Dans le cadre de l'OIT, les principaux instruments restent la pression morale et la pression des médias, qui sont censées garantir le respect des conventions sur une base de volontariat et de coopération. Quand on voit avec quelle énergie les représentants de gouvernements se battent pour éviter que les rapports nationaux de la commission d'application des normes ne contiennent des remarques négatives sur leur pays, il est évident que la pression morale joue un rôle capital.

Face à l'extension rapide des activités économiques internationales, le volontariat et la pression morale ont cependant des limites. D'un point de vue éthique, il convient donc de soutenir la mise sur pied, au niveau non seulement national mais aussi international, d'un système de récompenses en cas de respect et de pénalisations en cas de violation, les pénalisations pouvant prendre la forme de sanctions. II est non seulement indéfendable du point de vue éthique mais également contraire à la logique économique que des sanctions commerciales soient prononcées dans le cadre du GATT/OMC lorsque des pays exportateurs veulent favoriser artificiellement leurs produits en les vendant 
moins chers grâce à des subventions alors qu'il n'existe pas de sanctions similaires pour punir des avantages commerciaux obtenus en violant de façon caractérisée des droits fondamentaux des travailleurs. Pour des raisons de cohérence, il ne faut donc pas rejeter d'entrée le principe de sanctions commerciales en cas de violation de normes sociales minimales. ${ }^{13}$

L'idée de récompenser par des mesures positives les pays qui jouent le jeu est notamment appliquée par l'UE qui s'efforce de favoriser par le jeu des préférences douanières les exportations des pays en développement respectant les clauses sociales. Le problème de ce genre de mesures conditionnelles est qu'elles ne sont pas le fruit de négociations mais décidées unilatéralement.

\section{L'imposition de normes sociales minimales exige une collaboration à divers niveaux}

De même que l'éthique comprend une dimension individuelle, personnelle et sociale et que l'éthique économique englobe la sphère de la politique de réglementation, de l'entreprise et du consommateur, la régulation sociale du marché mondial requiert la participation de différenis niveaux d'action qui ne s'opposent pas. Dans cette perspective, les mécanismes de protection sociale doivent être étudiés selon le principe de l'égalité de traitement d'abord multilatéralement par I'OMC et l'OIT mais aussi dans les accords de commerce bilatéraux. Pour compléter ces mesures (et non pas pour les remplacer), les accords volontaires entre entreprises et organisations non gouvernementales (oeuvres d'entraide, organisations de commerce équitable, organisations de consommateurs) jouent un rôle important car ils sont plus facilement et plus rapidement applicables que des accords entre Etats et parce qu'ils suscitent une prise de conscience du consommateur. La montée rapide du commerce équitable et la clause sociale née en Suisse entre Migros et Del Monte (Philippines) à laquelle participent les oeuvres d'entraide en sont de bons exemples. II convient toutefois de faire une distinction entre clauses sociales relatives à des produits, à des entreprises, à des branches et à des pays ${ }^{14}$. II faut souvent que des approches au niveau de l'éthique individuelle et de l'entreprise jouent un rôle de pionnier pour que des réglementations politiques plus globales puissent recueillir une majorité.

13. C'est ce qui ressort des directives en matière de commerce international définies en 1980 par le "Gesprächskreises Kirche-Wirtschaft" dans le paragraphe sur les relations économiques avec les pays totalitaires et racistes (4.4), qui précise que les relations économiques sont à revoir si elles contraignent l'entreprise à adopter un comportement en contradiction flagrante avec des valeurs sociales et humaines fondamentales.

14. cf. à ce sujet Lanz, Ch.: Testfall Ananas. Die Sozialklausel Migros/Del Monte. Berne 1987; Stückelberger, Ch.: Sozialklauseln für fairen Welthandel. Neue Schritte mit der Sozialklausel Migros/Del Monte, in "Reformiertes Forum" 35, sept. 1994, 3-4; Egger, M.: Clause sociale. Un nouveau départ, Terre Nouvelle (Lausanne), nov.-déc. 1994, 22f; Report of the Second Migros-Del Monte Social Clause Monitoring Panel, 60 p., nov. 1995, Manille, Berne 